\title{
Analysis of the Welding Deformation of Resistance Spot Welding for Sheet Metal with Unequal Thickness*
}

\author{
Yuanxun $\mathrm{WANG}^{* *}$, Peng $\mathrm{ZHANG}^{* *}$, Ying $\mathrm{WU}^{* *}$ and Zhigang $\mathrm{HOU}^{* * *}$ \\ ${ }^{* *}$ School of Civil Engineering and Mechanics, Huazhong University of Science and Technology \\ Wuhan, 430074, China \\ E-mail: wangyuanxun@mail.hust.edu.cn \\ *** School of Mechanical and Electronic Engineering \& Automobile Engineering, Yantai University \\ Yantai, 264005, China
}

\begin{abstract}
Coupled electrical-thermal and thermo-elastic-plastic analysis were performed to analyze the transient thermal and mechanical behaviors of resistance spot welding (RSW) process of the mild steel sheet metals with unequal thickness. The thermal histories of the whole process and temperature distributions in the sheet metals were obtained through the electrical-thermal analysis. Different from the temperature field distribution of RSW with the same thickness mild steel sheet metals, the research showed that the center of the weld nugget of RSW with unequal thickness mild steel sheets leaned to the thicker sheet. It accords with the practical RSW process of the sheet metals with unequal thickness. The residual plastic strain distributions and the deformations of the sheets after welding were obtained through the thermo-elastic-plastic analyses. Due to the asymmetry of the temperature field distribution in the sheet metals with unequal thickness, the edges of the two mild steel sheets warped to the thinner one. The warpage deformation of the two sheets with the thickness $1.0 \mathrm{~mm}$ and $1.5 \mathrm{~mm}$ was $5 \mu \mathrm{m}$ after RSW. Detailed studies showed that the warpage deformation of the asymmetric sheet structure was resulted from the changed residual plastic strain.
\end{abstract}

Key words: Welding, Deformation, FEA, Temperature Field, Thermo-Elastic-Plasticity

\section{Introduction}

Welding sheet structures are widely used in many industries. For example, some important components such as automobile frame and body, truck cabin, railway vehicle and ship body are produced by various welding methods with profiled sheet materials and stamping parts. The weld-induced deformation has great influences on the quality of the welding sheet structures. Therefore, it is of great significance to research the welding deformation of the welding sheet structures and its control method to improve the quality of productions.

Resistance spot welding (RSW) process is widely used in sheet metal joining process due to its high speed, suitability for automation and inclusion in high-production assembly lines with other fabricating operations. It is a complex process in which coupled interactions exist between electrical, thermal, mechanical, metallurgical phenomena, and even surface behaviors. During RSW process, deformation, stress and strain will be generated and 
changed in the weldment due to the electrode force and Joule heating, and residual stress and strain will retain in the weldment after welding. Numerous researches of the mechanical features for such a complex process have been performed. These researches underwent all kinds of welding conditions and materials, using both theoretical and experimental methods ${ }^{(1)-(3)}$. It can be concluded from these researches that failure of spot weld is likely related to many parameters of the RSW process, e.g. residual stress, welding parameters, welding schedule, thickness, gap, nugget size, and material properties. These parameters also affect the fatigue life of the welded joint. Results of the relative researches ${ }^{(4)-(6)}$ show that the fatigue strength is mainly controlled by the residual stress, gap and stress concentration at the notch of nugget.

Recently, numerical method provides a powerful tool in studying these interactions, especially the finite element analysis (FEA) method, which can deal with nonlinear behaviors and complex boundary conditions. It has become the most important method for the analysis of RSW process ${ }^{(7)(8)}$. Tsai et al. ${ }^{(9)}$ developed a real-time control method in RSW and obtained direct correlations between nugget formation and expansion displacement of electrodes. Nied ${ }^{(10)}$ developed the first FEA model for the RSW process, investigated the effect of the geometry of electrode on workpiece and predicted the deformation and stresses. However, the developed model was restricted to elastic deformation, and could not calculate the contact areas at the electrode-workpiece and faying surface. Therefore, many researchers developed more sophisticated FEA models that taken the contact status, phase changing, and coupled field effects into the simulation of $\mathrm{RSW}^{(11)-(13)}$. The iterative method was employed to simulate the interactions between coupled electrical, thermal, and structural fields ${ }^{(14)(15)}$. In this method, the stress field and contact status were initially obtained from the thermo-mechanical analysis, and then the temperature field was obtained from the fully coupled electrical-thermal analysis based on the contact area at the electrode-workpiece interface and faying surface. The calculated temperature field was then passed back to the thermo-structural analysis to update the stress field and contact status. The iterative method can provide the temperature field, the electric potential field, the stress and strain distributions of the spot welding in one calculation, but the simulation of transient processes with such a methodology would probably require large amount of computing time. The objective of this paper is to develop a multi-coupled method to analyze the welding deformation of the RSW process, in order to reduce the computing time with the minimum loss of computing accuracy.

Based on the analysis of temperature field, thermo-elastic-plastic finite element analysis was attempted on the RSW process to analyze the distribution and change of the welding stress, strain and deformation in this paper.

\section{FEA modeling and governing equations}

\subsection{Model and Mesh}

For the solution of the welding deformation of the RSW process in this research an axisymmetric model was developed and solved using the FEA method based on ANSYS code. The two-dimensional axisymmetric model is illustrated schematically in Fig.1 where $x$ and $y$ represent the faying surface and the axisymmetric axis respectively. Its corresponding dimensions are $\mathrm{OE}=\mathrm{HI}=2.5 \mathrm{~mm}, \mathrm{OI}=\mathrm{EH}=15 \mathrm{~mm}, \mathrm{PA}=\mathrm{FG}=5 \mathrm{~mm}, \mathrm{~PB}=11 \mathrm{~mm}$, $\mathrm{AG}=18 \mathrm{~mm}, \mathrm{EF}=12.5 \mathrm{~mm}, \mathrm{ED}=3 \mathrm{~mm}, \mathrm{OP}=32 \mathrm{~mm}, \alpha=30^{\circ}$.

The model was meshed using three types of elements, as shown in Fig.2. The solid elements were employed to simulate the thermo-elastic-plastic behavior of the sheets and electrodes. The contact pair elements were employed to simulate the contact areas. There were three contact areas in the model. Contact area 1 and 2 represented the electrodeworkpiece interface and contact area 3 represented the faying surface. They were all assumed to be in contacts with two deformable surfaces, and these surfaces were allowed to 
undergo small sliding. In order to obtain reliable results, fine meshes were generated near these contact areas, while the meshes of other areas were relatively coarse.

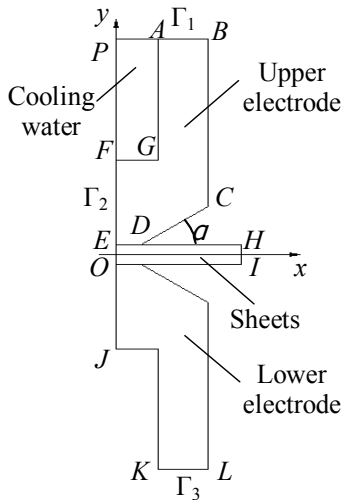

Fig. 1. Schematic diagram for the model of RSW

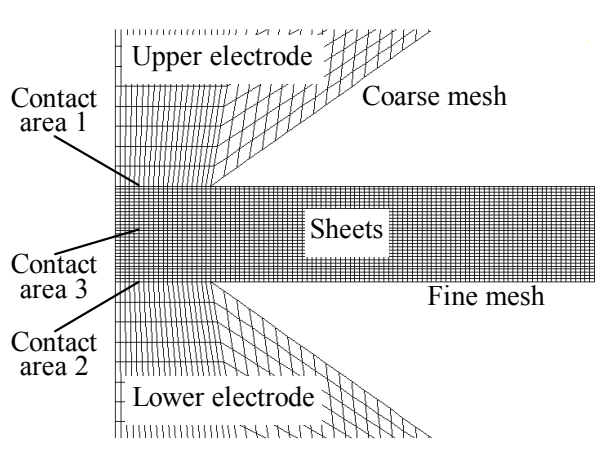

Fig. 2. Mesh generation of the developed model

\subsection{Governing equations and boundary conditions}

The distribution and change of the strain, especially the plastic strain, is very important to the analysis of residual stress and deformation of the RSW process. The welding residual stress is produced in welded joint as a result of plastic deformation caused by non-uniform thermal expansion and contraction in the RSW process. So the analysis of welding temperature field is very important and necessary for the welding deformation analysis, but it is a non-linear transient heat exchange problem. The governing equation for axisymmetric transient thermal analysis is given by the Fourier Law and the Energy Conservation Theory,

$$
\rho c \frac{\partial T}{\partial t}=\frac{\partial}{\partial x}\left(k_{x} \frac{\partial T}{\partial x}\right)+\frac{\partial}{\partial y}\left(k_{y} \frac{\partial T}{\partial y}\right)+\frac{\partial}{\partial z}\left(k_{z} \frac{\partial T}{\partial z}\right)+q_{v}
$$

where $\rho$ is the density of the material, $c$ is the specific heat capacity of the material, $T$ is the temperature, $t$ is the time course, $k_{x}, k_{y}, k_{z}$ are the thermal conductivity in three directions, $q_{v}$ is the rate of internal heat generation. For isotropic material, $k_{x}=k_{y}=k_{z}=k$. The thermal boundary conditions of the welding temperature field can be decomposed from the nonlinear isotropic Fourier thermal flux density and heat exchange. The thermal flux density shows the intensity of the welding thermal source, and the surface heat exchange presents the heat convection and heat radiation with the ambience. On the boundary surface, it is,

$$
\begin{array}{ll}
k_{n} \frac{\partial T}{\partial n}=q_{s}(x, y, z, t) & \text { (for thermal flux density boundary } S \text { ) } \\
k_{n} \frac{\partial T}{\partial n}=h\left(T_{a}-T_{s}\right) & \text { (for heat convection boundary } S c) \\
k_{n} \frac{\partial T}{\partial n}=\kappa\left(T_{r}-T_{s}\right) & \text { (for heat radiation boundary } S r \text { ) }
\end{array}
$$

in which, $n$ is the outward normal to the surface, $k_{n}$ is the thermal conductivity through the boundary normal, $q_{s}$ is the thermal flux through the boundary surface, $h$ is the heat convection coefficient, $T_{a}$ is the ambience temperature, $\kappa$ is heat radiation coefficient, $T_{r}$ is the radiation object temperature, $T_{s}$ is the boundary temperature.

To solve the nonlinear partial differential Eq.(1), the functional analysis can be established as, 


$$
\begin{aligned}
\Pi= & \int_{V} \frac{1}{2}\left\{k_{x}\left(\frac{\partial T}{\partial x}\right)^{2}+k_{y}\left(\frac{\partial T}{\partial y}\right)^{2}+k_{z}\left(\frac{\partial T}{\partial z}\right)^{2}\right\} \mathrm{d} V-\int_{S_{c}} h\left(T_{a} T_{s}-\frac{1}{2} T_{s}^{2}\right) \mathrm{d} S \\
& -\int_{S_{r}} \varepsilon f \sigma\left(T_{r}^{4} T_{s}-\frac{1}{5} T_{s}^{5}\right) \mathrm{d} S-\int_{S_{2}} T_{s} q_{s} \mathrm{~d} S-\int_{V} T q_{v} \mathrm{~d} V
\end{aligned}
$$

where $T$ is the inner temperature of the object, $T_{s}$ is the surface boundary temperature. Using the variation principle, function (3) can be obtained as,

$$
\delta \Pi=0
$$

that is

$$
\begin{aligned}
& \int_{V} T^{\prime T} \bar{K} T^{\prime} \mathrm{d} V-\int_{S_{c}} h\left(T_{a}-T_{s}\right) \delta T_{s} \mathrm{~d} S \\
& -\int_{S_{r}} \kappa\left(T_{r}-T_{s}\right) \delta T_{s} \mathrm{~d} S-\int_{S_{2}} q_{s} \delta T_{s} \mathrm{~d} S-\int_{V} q_{v} \delta T \mathrm{~d} V=0
\end{aligned}
$$

where,

$$
\begin{aligned}
T^{\prime} & =\left(\begin{array}{lll}
\frac{\partial T}{\partial x} & \frac{\partial T}{\partial y} & \frac{\partial T}{\partial z}
\end{array}\right)^{T} \\
\bar{K} & =\left[\begin{array}{ccc}
k_{x} & 0 & 0 \\
0 & k_{y} & 0 \\
0 & 0 & k_{z}
\end{array}\right]
\end{aligned}
$$

$\bar{K}$ is the thermal conductivity matrix.

For the FEA equation, the temperature field is dispersed into $n$ elements, then, the variation Eq.(4) can be expressed as,

$$
\delta \Pi=\sum_{1}^{n} \delta \Pi_{e}=0
$$

where, $\delta \Pi_{e}$ is the variation equation for each element.

Let the element shape function matrix be $N$, the element inner temperature can be expressed as,

$$
T=N T^{e}
$$

where, $T_{e}$ is the element node temperature matrix. The derivative of $T$ can be expressed as,

$$
T^{\prime}=B T^{e}
$$

where, $B=\left(\begin{array}{lll}N_{x}^{\prime} & N_{y}^{\prime} & N_{z}^{\prime}\end{array}\right)^{T}$. From Eq.(5), one obtains,

$$
\begin{gathered}
\sum_{1}^{n} \delta T^{e T} \int_{V_{e}} B^{T} \bar{K} B \mathrm{~d} V T^{e}+\sum_{1}^{r} \delta T^{e T} \int_{S_{c}} N^{T} h N \mathrm{~d} S T^{e}+\sum_{1}^{p} \delta T^{e T} \int_{S_{r}} N^{T} \kappa N \mathrm{~d} S T^{e} \\
=\sum_{1}^{r} \delta T^{e T} \int_{S_{c}} N^{T} h T_{a} \mathrm{~d} S+\sum_{1}^{p} \delta T^{e T} \int_{S_{r}} N^{T} \kappa T_{r} \mathrm{~d} S \\
\quad+\sum_{1}^{t} \delta T^{e T} \int_{S_{2}} N^{T} q_{s} \mathrm{~d} S+\sum_{1}^{n} \delta T^{e T} \int_{V_{e}} N^{T} q_{v} \mathrm{~d} V \\
\text { Let } K_{T}^{e}=\int_{V_{e}} B^{T} \bar{K} B \mathrm{~d} V, \quad K_{c}^{e}=\int_{S_{c}} N^{T} h N \mathrm{~d} S, \quad K_{r}^{e}=\int_{S_{r}} N^{T} \kappa N \mathrm{~d} S, \quad C_{T}^{e}=\int_{V_{e}} N^{T} C N \mathrm{~d} V \\
R_{B}^{e}=\int_{V_{e}} N^{T} q_{s h} \mathrm{~d} V, \quad R_{c}^{e}=\int_{S_{c}} N^{T} h T_{a} \mathrm{~d} S, R_{r}^{e}=\int_{S_{r}} N^{T} \kappa T_{r} \mathrm{~d} S, R_{S}^{e}=\int_{S_{2}} N^{T} q_{s} \mathrm{~d} S,
\end{gathered}
$$

where, $\boldsymbol{K}_{T}^{e}$ is the element thermal conductivity matrix; $\boldsymbol{K}_{c}^{e}$ is the element heat convection matrix; $\boldsymbol{K}_{r}^{e}$ is the element heat radiation matrix; $\boldsymbol{C}_{T}^{e}$ is the element specific heat capacity matrix; $\boldsymbol{R}_{B}^{e}$ is the node thermal flux vector of the inner thermal source; $\boldsymbol{R}_{c}^{e}$ is the node 
thermal flux vector of the heat convection; $\boldsymbol{R}_{r}^{e}$ is the node thermal flux vector of the heat radiation; $\boldsymbol{R}_{s}^{e}$ is the node thermal flux vector of the thermal conductivity. Let $C_{T}$ be the general specific heat capacity matrix, $K$ be the general thermal conductivity matrix, $P$ be the general thermal flux vector, the FEA equation of the transient temperature field can be obtained as,

$$
C_{T} \dot{T}+K T=P
$$

The governing equation of the electrical analysis is,

$$
\frac{\partial}{\partial r}\left(C_{e} \frac{\partial \varphi}{\partial r}\right)+\frac{C_{e}}{r} \frac{\partial \varphi}{\partial r}+\frac{\partial}{\partial z}\left(C_{e} \frac{\partial \varphi}{\partial z}\right)=0
$$

in which, $C_{e}$ is the electrical conductivity, $\Phi$ is the electrical potential.

The coupled electrical-thermal problem is solved by the following matrix equation:

$$
\left[\begin{array}{cc}
{\left[C^{t}\right]} & {[0]} \\
{[0]} & {[0]}
\end{array}\right]\left\{\begin{array}{c}
\{\dot{T}\} \\
\{\dot{V}\}
\end{array}\right\}+\left[\begin{array}{cc}
{\left[K^{t}\right]} & {[0]} \\
{[0]} & {\left[K^{v}\right]}
\end{array}\right]\left\{\begin{array}{l}
\{T\} \\
\{V\}
\end{array}\right\}=\left\{\begin{array}{c}
\{Q\} \\
\{I\}
\end{array}\right\}
$$

where, $\left[C^{t}\right]$ is the thermal specific heat matrix; $\left[K^{t}\right]$ is the thermal conductivity matrix; $\left[K^{v}\right]$ is the electric coefficient matrix; $\{T\}$ is the temperature vector; $\{V\}$ is the electric potential vector; $\{Q\}$ is the heat flow vector; $\{I\}$ is the current vector.

For the structural analysis, the stress equilibrium equation is given by,

$$
\nabla \sigma(r, t)+b(r, t)=0
$$

where, $\sigma$ is the stress, $b$ is the body force, $r$ is the coordinate vector.

The constructive equations of the materials based on thermo-elastic-plastic theory are given by,

$$
\begin{aligned}
& \mathrm{d}\{\sigma\}=[\boldsymbol{D}] \mathrm{d}\{\varepsilon\}-\{\boldsymbol{C}\} \mathrm{d} \boldsymbol{T} \\
& \{C\}=-\left[D^{e}\right]\left(\{\alpha\}+\frac{\partial\left[D^{e}\right]^{-1}}{\partial T}\{\sigma\}\right)
\end{aligned}
$$

where, $\{\sigma\}$ is the stress vector; $\{\varepsilon\}$ is the strain vector; $\{\alpha\}$ is the coefficient of thermal expansion; $[D]$ is the elastic-plastic matrix: $[D]=\left[D^{e}\right]$ in the elastic area, while $[D]=$ $\left[D^{e}\right]-\left[\boldsymbol{D}^{p}\right]$ in the plastic area. $\left[D^{e}\right]$ is the elastic matrix and $\left[\boldsymbol{D}^{p}\right]$ the plastic matrix.

The following boundary conditions are specified on the surface of $\Gamma 1, \Gamma 2$ and $\Gamma 3$ (see Fig. 1),

- $\Gamma 1(\mathrm{AB}): \sigma_{y}=-q$, where $q$ is the uniform pressure which can be determined according to the electrode force and the section area of the electrode.

- Г2(FEOJ): $U x=0$, where $U_{x}$ is the displacement of $\mathrm{x}$-direction.

- $\Gamma 3(\mathrm{KL}): U_{y}=0$, where $U_{y}$ is the displacement of y-direction.

\subsection{Welding parameters and material properties}

The welding parameters used in this analysis are, welding current $12.2 \mathrm{kA}$ ( $50 \mathrm{~Hz}$ sine wave AC), weld time $0.26 \mathrm{~s}$ ( 13 cycles), electrode force $3 \mathrm{kN}$, and hold time $0.06 \mathrm{~s}$ ( 3 cycles). Because the materials are subjected to a wide range of temperature, most of the material properties are considered as temperature dependent. The most important property in the analysis of the RSW process is the contact resistivity of faying surface. The contact resistivity is a dependent function on contact pressure, temperature, and average yield strength of two contact materials. Reference (16) pointed out that the contact resistance 
decreases as the contact pressure increases. Using a curve fitting procedure, Ref. (17) developed an empirical model for establishing the desired relationship of contact resistance against pressure and temperature. During the RSW process, the contact resistivity distribution influences the current density pattern, which affects the temperature field through Joule heating; while the temperature field then influences the mechanical pressure distribution through thermal expansion, related to the interface resistivity. Therefore, this is a highly non-linear problem involving the complex interaction of thermal, electrical and mechanical phenomena. To simplify the problem, many researchers took the contact resistivity as a function of temperature ${ }^{(11)(14)}$. This simplification is reasonable since, firstly, the load is constant in a specified RSW process; secondly, the yield strength of the materials, which determines the contact status in the contact area, is essentially influenced by temperature. With this simplification, the computing time can be reduced greatly. Therefore, in this research, the temperature dependent contact resistance was imposed at the faying surface.

The mechanical, electrical and thermal properties of both the copper electrode and mild steel sheet workpiece are shown in Fig.3.

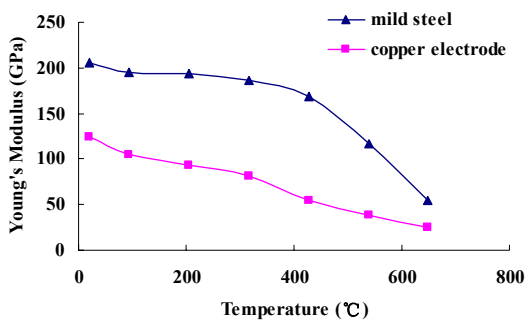

(a) Young's Modulus

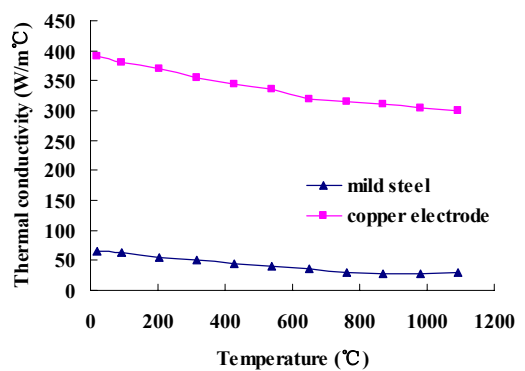

(c) thermal conductivity



(b) coefficient of thermal expansion

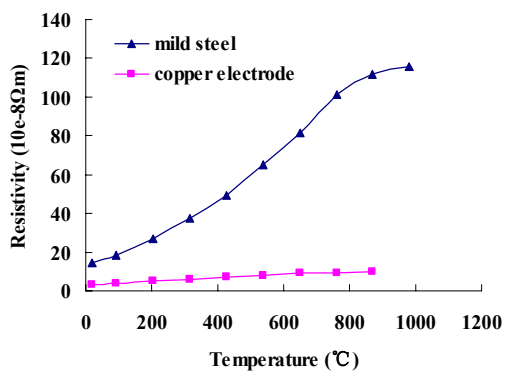

(d) resistivity

Fig.3 Mechanical, electrical and thermal properties of the mild steel and copper electrode

\section{Thermo-Elastic-Plastic Analysis}

The developing process of the welding residual stress and deformation of the RSW can be dynamically simulated through thermo-elastic-plastic analysis based on the FEA of the temperature field. The temperature fields and its changing of the sheet metal RSW have been obtained and well discussed through the coupled electrical-thermal analysis in Ref. (7) and Ref. (8). But the researches were limited at the RSW with the same thickness sheet metal. Figure 4 shows the temperature distribution of the RSW with unequal thickness mild steel sheets at the time of the nugget forming. Figure 5 shows the temperature changing courses at the center of the weld nugget, the center of the upper electrode and the lower electrode. 


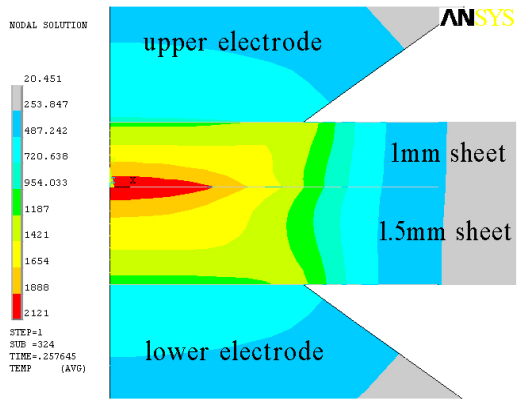

Fig.4 Temperature distributions of the RSW process

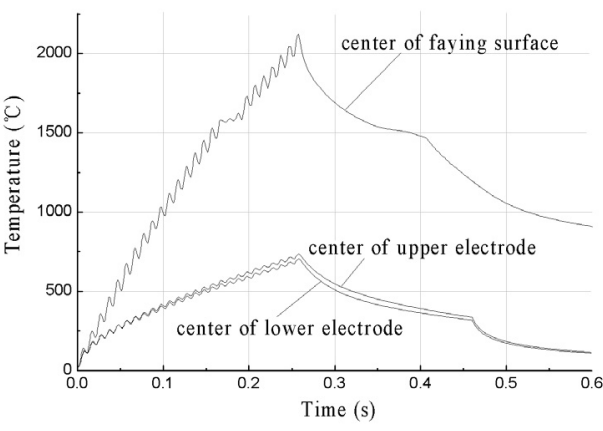

Fig.5 Temperature histories of the RSW process

Different from the temperature field distribution of RSW with the same thickness mild steel sheet metals, the research shows that the center of the weld nugget of RSW with unequal thickness mild steel sheets leans to the thicker workpiece; for more thermal is produced and less being given out in the thicker workpiece. Because the faying surface of the two mild steel sheet workpieces is further from the electrode acting on the thicker workpiece and therefore the resistance is bigger.

For the thermo-elastic-plastic analysis, some hypotheses are cited. The mechanical properties, stress and strain related with the welding temperature are linearly changed in a small time increment. Elastic stress, plastic stress and temperature stress are separable. Strain stiffening is occurred in the plastic field and obeys the Rheology's theory. The Mises Yield Criterion is used for the material yield strength. The welding thermo-elastic-plastic analysis is constructed by strain-displacement relationship or compatibility condition; stress-strain relationship or constitutive relationship; equilibrium condition and boundary conditions. The constitutive equations of the material in the temperature field can be written as eq. (16).

The temperature field analysis with the coupled electrical-thermal analysis showed that the center of the weld nugget of the RSW with unequal thickness mild steel sheets leaned to the thicker workpiece, for the asymmetry of the structure, the temperature field distribution was asymmetric too. Loading the temperature field at the corresponding time as the node body load, the structure analysis showed that the deformation of the RSW with unequal thickness mild steel sheets was also asymmetric. Figure 6 shows the welding deformation of the RSW of two mild steel sheets with the thickness $1.0 \mathrm{~mm}$ and $1.5 \mathrm{~mm}$ in which the dashed is the outline before deformation. Simulating analyses showed that the edges of the two mild steel sheets warped to the thinner one due to the structure asymmetry. Taking the two mild steel sheets as an integrated structure after welding, the edge's warpage deformation could be expressed with the normal displacement of the edge of the mild steel sheet. The warpage deformation of the two mild steel sheets with the thickness $1.0 \mathrm{~mm}$ and $1.5 \mathrm{~mm}$ was $5 \mu \mathrm{m}$ after RSW.
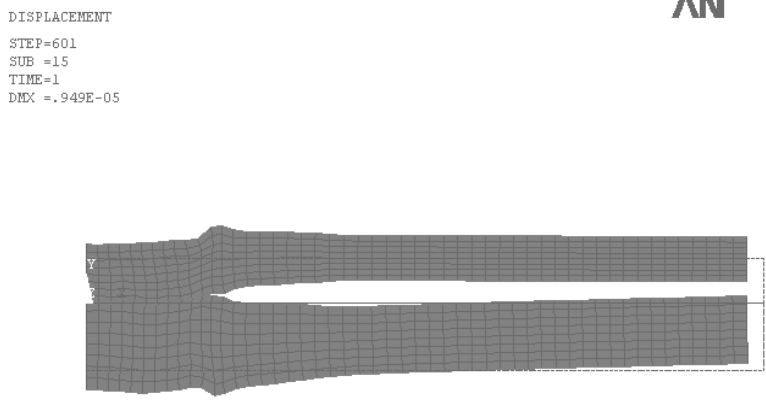

Fig.6 The welding deformation of the mild steel sheet with unequal thickness after RSW (amplified by10 times) 

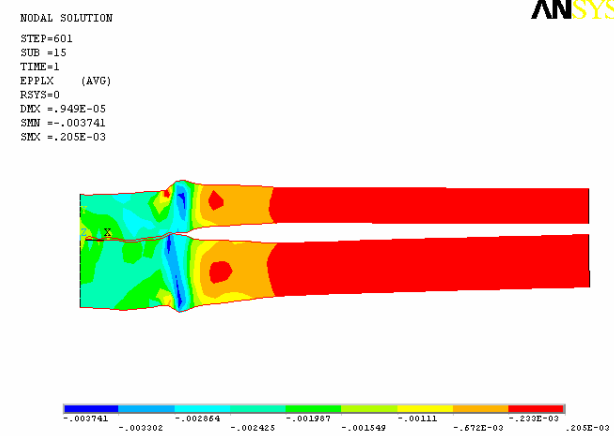

Fig.7 The residual plastic strain of the mild steel sheet with unequal thickness after RSW (amplified by10 times)

The asymmetric deformation is produced by the asymmetric residual plastic strain. Figure 7 shows the distribution of the residual plastic strain of the mild steel sheet with unequal thickness after RSW. It shows that the distributions of the residual plastic strain in the two mild steel sheets are different. The locations of the maximum residual plastic strain in the thicker sheet and the thinner one are different, and the radius of the distribution of the residual plastic strain in the thicker mild steel sheet is less than that in the thinner one. To study the impact of the residual plastic strain on the welding deformation, add the residual plastic strains of the nodes at the same thickness as its general residual plastic strain, it is different at the different thickness. Figure 8 shows the distributions of the residual plastic strain of the two mild steel sheets with unequal thickness.

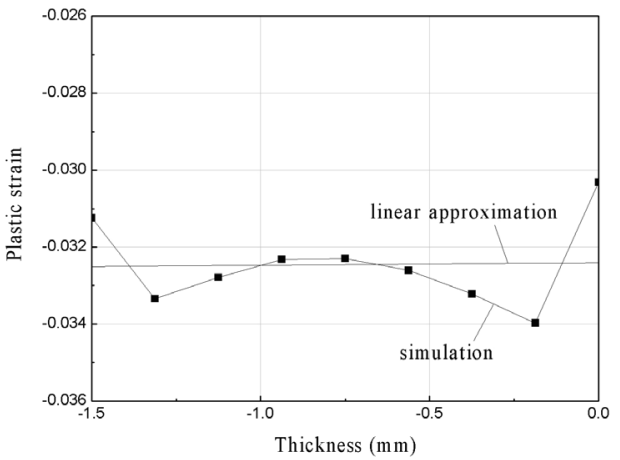

(a) the thicker mild steel sheet

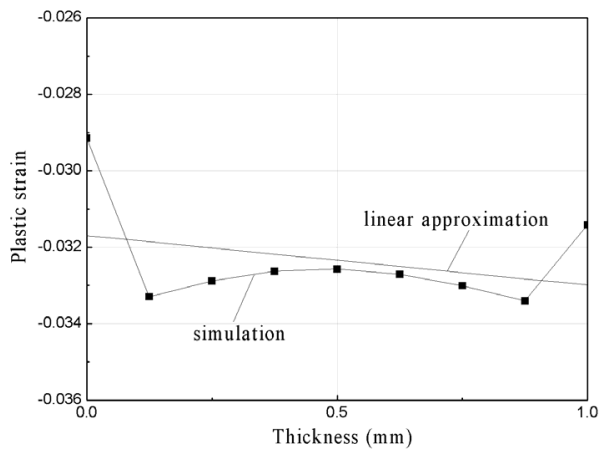

(b) the thinner mild steel sheet

Fig. 8 The distribution of residual plastic strain of the mild steel sheet

The linear approximation of the residual plastic strain shows that the distribution of the residual plastic strain in the thicker mild steel sheet is approximately uniform along with the thickness of the sheet. And the linear approximation curve is an approximate level beeline (see Fig.8(a)). But the distribution of the residual plastic strain in the thinner mild steel sheet is linearly changed along with the thickness of the sheet, it is smaller when near the faying surface and bigger on the other side (see Fig.8(b)). For the changed residual plastic strain, the warpage deformation of the sheet structure is produced.

\section{Conclusions}

Coupled electrical-thermal and thermo-elastic-plastic analyses were performed to analyze the transient thermal and mechanical behaviors of RSW process of the mild steel sheet metals with unequal thickness.

The thermal histories of the whole process and temperature distributions in the sheet metals were obtained through the electrical-thermal analysis. Different from the temperature 
field distribution of RSW with the same thickness sheet metals, the research showed that the center of the weld nugget of RSW with unequal thickness mild steel sheets leaned to the thicker sheet.

The distributions of residual plastic strain and the welding deformations of the sheets after welding were obtained through the thermo-elastic-plastic analyses. Due to the asymmetry of the temperature field distribution in the sheet metals with unequal thickness, the edges of the two sheets warped to the thinner one. The warpage deformation of the two sheets with the thickness $1.0 \mathrm{~mm}$ and $1.5 \mathrm{~mm}$ was $5 \mu \mathrm{m}$ after RSW. Detailed studies showed that the warpage deformation of the asymmetric sheet structure was produced due to the changed residual plastic strain.

\section{Acknowledgements}

The authors are grateful for the financial supported by Chinese Universities Scientific Fund and National Natural Science Foundation of China (11072083).

\section{References}

(1) Lin, S.H., Pan, J., Tyan, T. and Prasad, P., A general failure criterion for spot welds under combined loading conditions, International Journal of Solids and Structures, Vol.40, No.21 (2003), pp.5539-5564.

(2) Chao, Y.J., Ultimate strength and failure mechanism of resistance spot weld subjected to tensile, shear, or combined tensile/shear loads, Transaction of the ASME, Journal of Engineering Materials and Technology, Vol.125, No.2 (2003), pp.125-132.

(3) Deng, X., Chen, W. and Shi, G.., Three-dimensional finite element analysis of the mechanical behavior of spot welds, Finite Elements in Analysis and Design, Vol.35, No.1 (2000), pp.17-39.

(4) Adib, H., Gilgert, J. and Pluvinage, G., Fatigue life duration prediction for welded spots by volumetric method, International Journal of Fatigue, Vol.26, No.1 (2004), pp.81-94.

(5) Pan, N. and Sheppard, S., Spot welds fatigue life prediction with cyclic strain range, International Journal of Fatigue, Vol.24, No.5(2002), pp.519-528.

(6) Kang, H., Barkey, M.E. and Lee, Y., Evaluation of multiaxial spot weld fatigue parameters for proportional loading, International Journal of Fatigue, Vol.22, No.8(2000), pp.691-702.

(7) Hou, Z.G., Wang, Y.X., Li, C.Z. and Chen, C.Y., A multi-coupled finite element analysis of resistance spot welding process, Acta Mechanica Solida Sinica, Vol.19, No.1 (2006), pp.86-94.

(8) Hou, Z.G., Kim, I.S., Wang, Y.X., Li, C.Z. and Chen, C.Y., Finite element analysis for the mechanical features of resistance spot welding process, Journal of Materials Processing Technology, Vol.185, No.1-3(2007), pp.160-165.

(9) Tsai, C.L., Dai, W.L., Dickinson, D.W. and Papritan, J.C., Analysis and development of a real-time control methodology in resistance spot welding, Welding Journal, Vol.70, No.12 (1991), pp.339s-351s.

(10) Nied, H.A., The finite element modeling of the resistance spot welding process, Welding Journal, Vol.63, No.4(1984), pp.123s-132s.

(11) Cho, H.S., Cho, Y. J., A study of the thermal behavior in resistance spot welds, Welding Journal, Vol.68, No.6(1989), pp.236s-244s.

(12) Browne, D.J., Chandler, H.W., Evans, J.T., Wen, J., Computer simulation of resistance spot welding in aluminum: part I, Welding Journal, Vol.74, No.10(1995), pp.339s-344s.

(13) Yeung, K.S., Thornton, P.H., Transient thermal analysis of spot welding electrodes, Welding Journal, Vol.78, No.1(1999), pp.1s-6s.

(14) Khan, J.A., Xu, L., Chao, Y., Prediction of nugget development during resistance spot welding using coupled thermal-electrical-mechanical model, Science and Technology of Welding and Joining, Vol.4, No.4(1999), pp.201-207.

(15) Chang, B.H., Li, M.V., Zhou, Y., Comparative study of small scale and 'large scale' resistance spot welding, Science and Technology of Welding and Joining, Vol.6, No.5 (2001), pp.273-280.

(16) Vogler, M., Sheppard, S., Electrical contact resistance under high loads and elevated temperatures, Welding Journal, Vol.72, No.6(1993), pp.231s-238s.

(17) Babu, S.S., Santella, M.L., Feng, Z., Riemer, B.W., Cohron, J. W., Empirical model of effects of pressure and temperature on electrical contact resistance of metals, Science and Technology of Welding and Joining, Vol.6, No.3 (2001), pp.126-132. 